See the related article beginning on page 463 .

\title{
Insulin's effect on glucose production: direct or indirect?
}

\author{
Eugene J. Barrett \\ Department of Internal Medicine, University of Virginia Health Sciences Center, \\ Charlottesville, Virginia, USA \\ J. Clin. Invest. 111:434-435 (2003). doi:10.1172/JCI200317881.
}

Insulin's effects on glucose homeostasis have been studied as extensively as any area of metabolic regulation. It is therefore particularly exciting when new technologies allow a fresh look at older, well-studied but unresolved issues. This excitement is heightened when information from new approaches challenges accepted paradigms. Recent studies have proved that tissue-specific knockout mice provide such an approach. For example, tissue-specific deletion of the insulin receptor in skeletal muscle yields a mouse (the muscle-specific insulin receptor knockout or MIRKO) with a smaller musculature but with normal fasting and postchallenge glucose and insulin concentrations (1). This was quite unexpected inasmuch as physiologic studies in humans had indicated that greater than $80 \%$ of insulin-mediated glucose disposal occurred in skeletal muscle. Why then, despite severe skeletal muscle insulin-resistance, was overall body glucose homeostasis not impaired? Recognizing that developmental compensation for the absence of insulin receptors in muscles might have occurred, investigators examined downstream signaling molecules of the insulin receptor pathway as well as the

\footnotetext{
Address correspondence to: Eugene J. Barrett, Department of Internal Medicine, University of Virginia Health Sciences Center, Box 801410, Charlottesville, Virginia 22908, USA.

Phone: (804) 924-1175; Fax: (804) 924-1284;

E-mail: ejb8x@virginia.edu.

Conflict of interest: The author has declared that no conflict of interest exists.

Nonstandard abbreviations used: musclespecific insulin receptor knockout (MIRKO); liver-specific insulin receptor knockout

(LIRKO); hepatic glucose production (HGP).
}

amount of IGF-1 receptor in muscle. No compensatory processes were identified. By default they concluded that tissues other than muscle, at least in the mouse, were more important in the maintenance of normal glucose metabolism. Interestingly, selective disruption of the Glut-4 transporter in muscle produced insulin resistance as well as glucose intolerance in some mice. Again however, this did not have as profound metabolic consequences as might be expected (2). Notably, deletion of Glut-4 in adipose also produced insulin resistance in muscle (and liver), however the mechanism for these secondary effects has not been defined (3).

\section{Other knockouts raise more questions}

Subsequent characterization of the liver-specific insulin receptor knockout (LIRKO) mouse by investigators at the Joslin Clinic yielded a mouse with a small liver, extreme hyperinsulinemia with near-normal fasting glucose, and normal rates of basal glucose turnover (4). Following a glucose challenge, LIRKO animals demonstrated severe glucose intolerance. In addition, in this animal, synthesis of several hepatic proteins controlled by insulin (e.g., glucokinase and albumin) was diminished while messenger RNAs coding for gluconeogenic enzymes were elevated. Though basal glucose production rates were normal in the LIRKO animals, low-dose insulin infusion did not suppress glucose production demonstrating hepatic as well as peripheral insulin resistance. This animal is not a longterm stable model as progressive hepatic dysfunction develops with age and by six months the animal presents with fasting hypoglycemia, elevated serum transaminase concentrations, and histologic evidence of hepatic steatosis.

Continuing this tour de force exploitation of the Cre-lox technology, the Joslin group reported that tissuespecific deletion of the insulin receptor in pancreatic $\beta$ cells led to a defect in first-phase insulin secretion (not unlike that seen in type 2 diabetes) and progressive glucose intolerance as the animals aged (5). The glucose intolerance was particularly striking in the female mice. This is strong evidence of a previously unanticipated and important role for insulin receptors in islet cells in the normal regulation of insulin secretion. Subsequently, they demonstrated that deletion of insulin receptors specifically in the brain produced mild hyperphagia, obesity, and with time, mild insulin resistance (6).

\section{LIRKO confronts \\ physiologic questions}

Beyond characterization of the phenotype, these models have begun to help in sorting through more detailed aspects of insulin action (7). In this issue of the JCI, Fisher and Kahn use the LIRKO mouse to test the hypothesis that insulin principally regulates hepatic glucose production (HGP) via indirect action in extrahepatic tissues (8). More specifically, very careful studies from several laboratories had suggested that insulin's suppression of lipolysis in adipose tissue lowered free fatty acid concentrations and this, in turn, significantly contributes to the decline in HGP (8-14). In these studies, in dogs and humans, it was not possible to selectively block insulin's action on the liver while allowing full expression of its effects in peripheral tissues. However, carefully designed experiments in which portal or peripheral insulin concentrations were selectively and independently varied convincingly demonstrated an indirect or peripheral effect of insulin to lower hepatic glucose production (13).

The LIRKO mouse provides an interesting opportunity to test insulin's effects on HGP in a setting where direct hepatic effects of insulin appear to be fully blocked. The caveat is, of course, whether insulin's peripheral actions in this severely insulin-resistant animal 
mimic those seen in more normal physiologic settings. The authors demonstrate that even very high insulin concentrations (well beyond the physiologic range) are ineffective in suppressing HGP in the LIRKO mice while fully suppressing HGP in the control animals (8). Serum free fatty acids are similar in the LIRKO and control animals and decline in both with insulin. Plasma albumin levels in the LIRKO animals however are only onehalf those in the controls. Whether the liver is exposed to similar amounts of adipose tissue-derived fatty acids in the LIRKO and control animals was not ascertained. As a result, it is difficult to exclude the possibility that differences in fatty acid availability within the liver might still contribute to the lack of suppression of hepatic glucose production in the LIRKO mice. The profound insulin resistance of the LIRKO mice may have been in part attributable to the decreased hepatic insulin clearance and receptor downregulation in peripheral tissues. Streptozotocin treatment may have diminished this marginally, but did not result in any evidence for a recovery of an indirect action of insulin on HGP in this model.

It would have been interesting to have measurements of amino acids, glycerol, and lactate and pyruvate in the LIRKO mice. The LIRKO livers have diminished glycogen stores and appear to rely entirely on gluconeogenesis for glucose production. At baseline, this is somewhat different than both the human and canine models where glycogenolysis accounts for $50-80 \%$ of hepatic glucose production. Inasmuch as the liver of the LIRKO mouse is only approximately one-half the size of control livers and as glucose production rates are comparable the single pass hepatic extraction of gluconeogenic substrates must be very high in LIRKO mice and peripheral effects of insulin to lower circulating concentrations of amino acids and glycerol might impact upon this source of carbon for glucose production. However, given the insulin resistance seen in the peripheral tissues to glucose disposal, it may be that a similar resistance extends to suppression of proteolysis, or glycerol production.

Although there was virtually no suppression of HGP in the LIRKO animal, these studies do not negate the demonstrated effect of peripheral insulin to decrease HGP in more physiologic settings where there is basal insulin activity operating on the liver. Indeed one surprise in the LIRKO model is that in the absence of any detectable insulin signaling, with gluconeogenesis dominant in liver and with low glycogen stores and available FFAs, these animals are apparently not ketotic or ketoacidotic and grow near to normal. Likely again, adaptive changes have occurred during development that counter the ketosis that might otherwise be anticipated. The nature of this adaptation is not defined. Such considerations serve to emphasize that although the LIRKO mouse provides an interesting and useful model, conclusions relating to the regulation of normal physiology based on studies of this animal must be laced with a dose of caution.

The last four decades of the 20th century witnessed the development of technologies like radioimmunoassay, isotopic turnover methods, the insulin-clamp method, in vivo NMR spectroscopy, and other tools to quantify the metabolism of glucose, fatty acids, and proteins in humans in vivo and define the dysregulation of these processes as occurs in diabetes, glucose intolerance, obesity, and other common metabolic disorders. These tools can now be coupled with genetic manipulations available specifically in murine species. Combined, these methods offer a powerful way to test specific hypotheses incisively. We have seen only the beginning of the exploitation of the power of these combined methodologies. We can anticipate that information gained from studies of the type reported in this issue of the JCI will continue to challenge our presumed understanding and lead to a very different integrated understanding of insulin's regulation of body metabolism than is available to us today.

1. Bruning, J.C., et al. 1998. A muscle-specific insulin receptor knockout exhibits features of the metabolic syndrome of NIDDM without altering glucose tolerance. Mol. Cell. 2:559-569.

2.Zisman, A., et al. 2000. Targeted disruption of the glucose transporter 4 selectively in muscle causes insulin resistance and glucose intolerance. Nat. Med. 6:924-928.

3. Abel, E.D., et al. 2001. Adipose-selective targeting of the GLUT4 gene impairs insulin action in muscle and liver. Nature. 409:729-733.

4. Michael, M.D., et al. 2000. Loss of insulin signaling in hepatocytes leads to severe insulin resistance and progressive hepatic dysfunction. Mol. Cell. 6:87-97.

5. Kulkarni, R.N., et al. 1999. Tissue-specific knockout of the insulin receptor in pancreatic beta cells creates an insulin secretory defect similar to that in type 2 diabetes. Cell. 96:329-339.

6. Bruning, J.C., et al. 2000. Role of brain insulin receptor in control of body weight and reproduction. Science. 289:2122-2125.

7. Wojtaszewski, J.F., et al. 1999. Exercise modulates postreceptor insulin signaling and glucose transport in muscle-specific insulin receptor knockou mice. J. Clin. Invest. 104:1257-1264.

8. Fisher, S.J., and Kahn, C.R. 2003. Insulin signaling is required for insulin's direct and indirect action on hepatic glucose production. J. Clin. Invest. 111:463-468. doi:10.1172/JCI200316426.

9. Ader, M., and Bergman, R.N. 1990. Peripheral effects of insulin dominate suppression of fasting hepatic glucose production. Am. J. Physiol. 245:E1020-E1032.

10. Rebrin, K., Steil, G.M., Getty, L., and Bergman, R.N. 1995. Free fatty acid as a link in the regulation of hepatic glucose output by peripheral insulin. Diabetes. 44:1038-1045.

11. Rebrin, K., Steil, G.M., Mittelman, S.D., and Bergman, R.N. 1996. Causal linkage between insulin suppression of lipolysis and suppression of liver glucose output in dogs. J. Clin. Invest. 98:741-749.

12. Lewis, G.F., Vranic, M., Harley, P., and Giacca, A 1997. Fatty acids mediate the acute extrahepatic effects of insulin on hepatic glucose production in humans. Diabetes. 46:1111-1119.

13. Sindelar, D.K., Balcom, J.H., Chu, C.A., Neal, D.W., and Cherrington, A.D. 1996. A comparison of the effects of selective increases in peripheral or portal insulin on hepatic glucose production in the conscious dog. Diabetes. 45:1594-1604.

14. Sindelar, D.K., et al. 1997. The role of fatty acids in mediating the effects of peripheral insulin on hepatic glucose production in the conscious dog Diabetes. 46:187-196 